\section{In der Windel sind Bifidobakterien gut, Clostridien schlecht}

\author{
Der Darm Neugeborener ist zunächst steril. Die nach der Geburt \\ einziehende intestinale Mikroflora entscheidet offensichtlich \\ darüber, ob sich bei Risikokindern später eine Atopie entwickelt.
}

D en Einfluss der mikrobiellen Darmbesiedelung bei Neugeborenen auf die spätere Atopie-Entwicklung nahm jetzt eine prospektive Studie unter die Lupe. Dazu untersuchte der finnische Wissenschaftler Kalliomäki die intestinale Mikroflora von 76 Kindern, die er aufgrund der Familienanamnese als stark Atopie-gefährdet einschätzte, im Alter von drei Wochen, drei Monaten und einem Jahr. Zeigte sich mit zwölf Monaten mindestens eine positive Pricktestreaktion, stufte er die Kinder als atopisch ein. Dies traf in $22(29 \%)$ der Fälle zu.

Im Alter von drei Wochen ergab sich ein deutlicher Zusammenhang zwischen der Zusammensetzung der Darmflora und der Entwicklung einer allergischen Disposition: Die kleinen Atopiker hatten zu diesem Zeitpunkt signifikant mehr Clostridienspezies $(\mathrm{p}=$ $0,04)$ und weniger Bifidobakterien $(\mathrm{p}=$ $0,11)$ im Stuhl als die Nicht-Atopiker.

Im Alter von drei Monaten waren die Unterschiede in der bakteriellen Darmbesiedlung zwischen den beiden Gruppen weitgehend verschwunden. Nicht-Atopiker hatten zu diesem Zeitpunkt aber etwas häufiger Hefen im Stuhl.

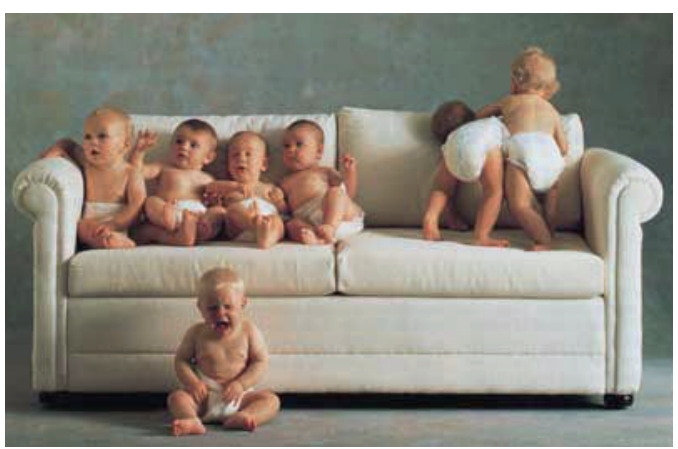

Die genauere Untersuchung des Windelinhalts gibt Aufschlüsse über die AtopiePathogenese.

\section{Fazit}

Die Besiedlung des Darmes Neugeborener mit unterschiedlichen Bakterien könnte über die Entwicklung einer Atopie (mit-)entscheiden. Die genauen Mechanismen sind noch unklar. Es wird
$M$ it Hilfe eines Fragebogens befragten Allergologen der Universität Nancy 33.110 Personen zu diversen allergischen Reaktionen u.a. auf Nahrungsmittel. 1.129 von ihnen wurden ein zweites Mal detailliert befragt, weil sich Hinweise auf eine Nahrungsmittel-Allergie ergeben hatten.

Es fand sich eine Prävalenz der Nahrungsmittel-Allergie von 3,24\%. Vermehrt betroffen waren Stadtbewohner, Frauen und Beschäftigte des Gesundheitswesens. 57\% der Nahrungsmittel-Allergiker hatten atopische Erkrankungen. Die NahrungsmittelAllergie bestand oft langfristig, bei 91\% der Betroffenen länger als sieben Jahre.

Die häufigsten Allergene stammten mit 14\% aus der Familie der Rosaceae, gefolgt von Gemüsen (9\%), Milch und Krustentieren mit jeweils 8\% und mit Naturlatex kreuzreagierenden Früchten (5\%). Weiter hatten 4\% eine Ei-Allergie, 3\% eine Nuss- und 1\% eine Erdnuss-Allergie. Eine Pollen-Sensibilisierung war signifikant korreliert mit dem Auftreten von Angioödem, Asthma, Rhinitis und Allergie gegen Früchte. Bestand eine Naturlatex-Allergie, so war eine Nahrungsmittel-Allergie viermal jedoch vermutet, dass bakterielle Zellbestandteile, z.B. Lipopolysaccharide, die Ausbildung einer physiologischen TH1-TH2-Zellbalance fördern. In weiteren Studien konnte der finnische Pädiater zeigen, dass die mütterliche Einnahme von Bifidobakterien in Schwangerschaft und Stillzeit die Atopie-Inzidenz bei Risikokindern deutlich senken konnte [wir berichteten im Allergo J 2001, 10: 244].

$b k$

Kalliomäki $\mathbf{M}$ et al. Distinct patterns of neonatal gut microflora in infants in whom atopy was and was not developing. J Allergy Clin Immunol 2001; 107: 129-34

\title{
Nahrungsmittelallergie unverhofft oft
}

\section{Zuverlässige Daten zur Häufigkeit von Nahrungsmittel-Allergien in der Allgemeinbevölkerung sind selten. Eine französische Studie liefert jetzt überraschende neue Erkenntnisse.}

häufiger. Bei Kindern unter sechs Jahren manifestierte sich die Nahrungsmittel-Allergie vorwiegend als atopisches Ekzem, bei Vier- bis Sechsjährigen als Asthma. Bei über Dreißigjährigen war die Hauptmanifestation der anaphylaktische Schock. Es fand sich ein signifikanter Zusammenhang zwischen dem Auftreten einer Anaphylaxie und der Einnahme von nichtsteroidalen Antiphlogistika bzw. Alkoholgenuss.

\section{Kommentar}

Der Wert dieser Studie wird eingeschränkt durch die Tatsache, dass es sich um eine Fragebogenaktion handelt. Dennoch ergeben sich für die Praxis wichtige Hinweise, so z.B. die erhöhte Prävalenz von Nahrungsmittel-Allergien bei Beschäftigten des Gesundheitswesens und der Zusammenhang zwischen Alkoholgenuss bzw. Einnahme nichtsteroidaler Antiphlogistika und dem Auftreten von schwerer Anaphylaxie. Dr. A. Niedermeier, München

Kanny G et al. Population study of food allergy in France. J Allergy Clin Immunol 2001; 108: 133-40 